\title{
NOTE SULL'ORDINE DEGLI ELEMENTI IN COPPIE DI VERBI ANTONIMI
}

\section{Premessa}

La riflessione sulle categorie e le proprietà legate al fenomeno dell'antonimia riguarda, in semantica, una serie di fatti senza dubbio interessanti e di notevole complessità. La definizione comune, tradizionale, di antonimia - in una certa misura anche generica - designa, in buona sostanza, l'antitesi tra elementi della lingua, tra parole, che hanno un "significato contrario"1. A partire da tale definizione, che richiama per altro il principio binario di opposizione che costituisce uno dei fondamenti strutturali delle lingue, vd. Lyons (1980: $294 \mathrm{sgg}$.), numerosi sono i modelli di analisi, le tassonomie e le tipologie elaborate per una migliore descrizione del fenomeno o di casi particolari del medesimo ${ }^{2}$. Si proporranno qui, in particolare, alcune brevi osservazioni sull'ordine in cui si presentano i termini che formano coppie di verbi antonimi (reciproci).

\section{Antonimi grammaticali e antonimi lessicali}

Tra i problemi legati alla descrizione dei caratteri propri dell'antonimia, in generale, sicuramente di un certo interesse è il tentativo di distinguere, in una data coppia di elementi di significato contrario, un termine "positivo" e un termine "negativo", un termine marcato e un termine non marcato. ${ }^{3}$ Dal punto di vista più propriamente morfologico, gli antonimi si sogliono dividere in antonimi legati da una relazione di tipo morfologico, cioè gli antonimi frutto di un processo di affissazione, derivazione $o$ altro che ne stabilisca l'opposizione (p.es. l'it. fare vs. disfare, possibile vs. impossibile), e antonimi privi di tale relazione (p.es. l'it. caldo vs. freddo, comperare

1 Pare esserci accordo, tra quanti si sono occupati del problema, sul fatto che l'antonimia riguardi gli elementi della lingua maggiormente legati alla dimensione lessicale, dunque soprattutto l'aggettivo e il nome (ma anche il verbo), piuttosto che la morfologia o la sintassi (quindi anche gli articoli, le preposizioni, il genere grammaticale etc.).

2 Si veda, in particolare, Stati (1977) e Dings (1986).

3 L'uso dei termini positivo vs. negativo non è legato qui alla valutazione di un certo obiettivo da raggiungere, obiettivo che può naturalmente variare a seconda delle circostanze o del soggetto che si prefigge di raggiungerlo, vd. Lewin (1961). Così, in un medesimo contesto, per una coppia di verbi reciproci come comperare e vendere, il comperare sarà positivo per la massaia che va fare alla spesa, il vendere positivo per il commerciante che offre la sua merce. Positivo e negativo potrebbe essere forse mutato in precedente e seguente, in riferimento al momento di svolgimento di due azioni, come anche in itivo e ventivo o progressivo e regressivo, in riferimento al crescere e al decrescere di una certa distanza o di una certa quantità. 
vs. vendere): i primi sono chiamati generalmente antonimi grammaticali o morfoseman-tici, i secondi antonimi lessicali o semantici, vd. Ducháček (1965: 56) ${ }^{4}$. Ancora in base ad una opposizione di tipo morfologico, questi elementi sono distinti anche in antonimi primari, che non presentano cioè marche derivative di segno negativo, privativo o altra relazione morfologica (p.es. comperare vs. vendere) e antonimi secondari, che al contrario ne presentano una o più di una (p.es. fare vs. disfare), vd. in particolare Geckeler (1979: 152 sgg.) e (1983). In una coppia di antonimi secondari (o grammaticali o morfosemantici) l'opposizione è dunque tra due elementi che possiedono la medesima base lessicale e che predicano, pertanto, la medesima qualità o azione, dove l'uno presenta la base da sola e l'altro la base con l'aggiunta di un modificatore (di segno negativo o privativo). Tra un elemento modificato e uno non modificato, sarà evidentemente l'elemento non modificato a costituire il termine "positivo" della coppia, il termine che afferma la presenza di una determinata qualità o lo svolgimento di una certa azione; sarà d'altra parte l'elemento modificato a predicare la negazione della qualità o dell'azione portata dalla semplice base lessicale e a costituire, quindi, il termine "negativo" della coppia ${ }^{5}$. Così tra due antonimi come fare e disfare, il verbo positivo è evidentemente fare, che descrive l'azione di 'eseguire, costruire, agire' (e altro ancora), mentre disfare predica l'azione contraria di 'distruggere, smantellare, scomporre'. Analogamente, tra due aggettivi come possibile e impossibile, il primo predicherà la presenza o la realtà della possibilità di una determinata circostanza o condizione, il secondo l'assenza di tale possibilità. Per questo tipo di antonimi, come si vede, il termine positivo risulta anche quello non marcato - diciamo basico o di partenza - mentre marcato risulta quello negativo.

Discorso più complesso, sul quale non ci soffermeremo, richiede il riconoscimento del termine positivo o negativo per gli antonimi che abbiamo definito primari (o lessicali o semantici), i quali, mettendo a confronto basi diverse, non consentono di distinguere un elemento morfologico che opponga un termine marcato ad un termine non marcato. In questo caso, la valutazione del rapporto tra i due antonimi va portata a livello più strettamente semantico, dove per esempio nella coppia di antonimi caldo e freddo la qualità predicata da entrambi gli aggettivi è il calore, così che caldo ne predica la presenza (risultando positivo, quindi non marcato) e freddo l'assenza (negativo, quindi marcato), vd. Geckeler (1979: 157-160).

Interessante è anche il caso dei lessemi che possiedono sia un antonimo primario che un antonimo secondario. Si tratta di aggettivi come attivo o mobile, ad esempio, che hanno tanto un antonimo grammaticale, rispettivamente inattivo e immobile,

4 Il legame tra questi antonimi è detto anche morpho-sémantique e sémantique da Klein (1975), mentre Lyons preferisce parlare, rispettivamente di morphologically related e morphologically unrelated.

$5 \mathrm{Ci}$ riferiamo, in particolare, a modificatori (tipicamente prefissi o prefissoidi, nelle nostre lingue) che esprimono una marca negativa o privativa, come per esempio gli italiani $a-/ a n-$, anti-, dis-, in-/im-: aerobico vs. an-aerobico, sociale vs. a-sociale (o anche anti-sociale), tossina vs. anti-tossina, pari vs. dis-pari, ordine vs. dis-ordine, credibile vs. in-credibile etc. 
quanto un antonimo lessicale, passivo e fisso ${ }^{6}$. Lo sbilanciamento di questo sistema a tre elementi è evidente e, aggiungo, piuttosto curioso: mentre infatti uno dei due termini in opposizione lessicale (attivo e mobile) possiede il suo antonimo grammaticale (inattivo e immobile), l'altro termine (passivo e fisso) non lo possiede ( ${ }^{*}$ apassivo, *antifisso, ${ }^{*}$ impassivo, ${ }^{*}$ controfisso o altro ${ }^{7}$ ). Molto stimolante sarebbe, a questo punto, non solo capire il motivo dell'assenza di antonimi grammaticali (secondari) per passivo e fisso, ma anche distinguere il significato e la funzione dei due antoni$\mathrm{mi}$ che si oppongono ad un unico termine, cioè passivo e inattivo rispetto ad attivo, e fisso e immobile rispetto a mobile ${ }^{8}$. Se infatti la lingua assegna, in generale, maggiore evidenza e rilievo alle cose, agli stati e alle azioni che necessitano di specificazioni più precise (con gli psicologi diremmo agli elementi che favoriscono un "comportamento adattivo" all'ambiente), dalla composizione di queste coppie di antonimi pare che la condizione di stato predicata da quattro aggettivi come inattivo e passivo, immobile e fisso, sia in generale più importante, o più utile da precisare, della condizione di moto predicata dai loro due contrari attivo e mobile 9 .

\section{Ordine degli elementi in coppie di verbi antonimi}

La questione del riconoscimento di un termine semanticamente positivo e di uno negativo all'interno di una coppia di antonimi - abbiamo brevemente visto il caso di due aggettivi come attivo e passivo, indicanti rispettivamente presenza e assenza di azione - trova un interessante riscontro nell'esame dell'ordine in cui si presentano, nella frase, due elementi verbali di significato contrario.

Consideriamo, dunque, alcune coppie di verbi antonimi (reciproci), che predicano prima una determinata azione e quindi l'inversione della stessa con il ritorno allo stato di partenza ${ }^{10}$. Il caso più trasparente, ancora una volta, è quello di elementi legati dal punto di vista morfologico. Si tratta, ad esempio, di verbi come dare,

6 Per opposizioni di questo tipo, Iliescu (1977) parla di antonimie linguistique 'antonimia linguistica' e di antonimie logique 'antonimia logica'. Analogo a quello di questi aggettivi è anche il caso di verbi come chiudere o fare, che hanno tanto antonimi grammaticali, con dischiudere e disfare, che antonimi lessicali, con aprire e distruggere, vd. anche Vicario (c.s.).

7 Il composto affisso è naturalmente formato dal prefisso ad- del latino, non dal privativo a-, mentre infisso vuol dire ancora 'fisso, fissato'.

8 Discutendo della relazione logica che si instaura tra gli aggettivi tedeschi sparsam 'parsimonioso', unsparsam 'spendaccione' (antonimo grammaticale) e verschwenderisch ancora 'spendaccione' (antonimo lessicale), Iliescu (1977: 157) giustamente osserva che "le plus souvent de telles paires opposables binaires contradictoires ne sont pas équivalents du point de vue logique des paires correspondantes dont le terme négatif $B$ est représenté par un lexème autre que $A$ ".

9 Ciò risuita almeno in parte sorprendente, a prima vista, se si pensa al cospicuo numero di verbi che l'italiano (ma anche altre lingue, naturalmente) riserva alla descrizione di diversi tipi di moto rispetto alla relativa povertà di tipi che descrivono condizioni di stato, una distribuzione che capovolge la gerarchia tra stato e moto evidenziata dagli aggettivi qui presi in considerazione.

10 Sui diversi rapporti di opposizione antonimica, ivi compresa la reciprocità (o inversione), vd. ancora Lyons (1971: 608-621). 
mandare, portare, spedire, che descrivono il moto proprio o figurato di un oggetto da $\mathrm{A}$ a $\mathrm{B}$, e dei loro reciproci ridare, rimandare, riportare, rispedire, che rappresentano il moto di senso contrario, di ritorno, da $\mathrm{B}$ ad $\mathrm{A}^{11}$ :

(1) a. Mi ha ridato il libro

b. Era forse il caso di rimandare la pratica all'Ufficio legale

c. Non può riportarmi l'automobile prima di domani sera

d. Hanno rispedito il plico al mittente

In questi casi, che vedono la funzione reversiva affidata al prefisso verbale $r i$, le frasi implicano, pur senza essere propriamente ellittiche, una prima azione (sottintesa, non espressa) che porta l'oggetto nella posizione dalla quale si predica il ritorno. Così, completare logicamente gli enunciati potrebbe portare a frasi di questo tipo:

(2) a. Gli ho dato il libro e lui me lo ha ridato

b. L'Ufficio legale ha mandato una pratica, che era forse il caso di rimandargli

c. Gli ho portato l'automobile, ma non può riportarmela prima di domani sera

d. Aveva regolarmente spedito il plico, che è stato rispedito al mittente

Negli esempi presentati in (2) la predicazione della seconda azione, quella di ritorno (posteriore), segue la predicazione dell'azione di andata (anteriore), rispettando l'ordine temporale di svolgimento delle stesse. Non è possibile predicare lo svolgimento di un'azione di ritorno, successiva dal punto di vista fattuale, senza il compiuto svolgimento dell'azione di andata:

(3) a. Gli ho dato (ieri) il libro e lui me lo ha ridato (oggi)

b. Mi ha ridato (oggi) il libro che gli ho dato (ieri)

c. *Gli ho dato (oggi) il libro e lui me lo ha ridato (ieri)

d. *Mi ha ridato (ieri) il libro che gli ho dato (oggi)

In (3a) gli avverbi di tempo ieri (anteriore) e oggi (posteriore) possono essere espressi o sottintesi, dando magari per noto il contesto temporale nel quale si sono svolte le azioni, ma non si può comunque collegare ieri (anteriore) con ridato (posteriore) o oggi (posteriore) con dato (anteriore), come figura in (3c). Lo stesso discorso vale anche se si inverte l'ordine delle proposizioni, e quindi dei verbi, come in (3b) $o$ (3d). Ancora più chiaro, se possibile, un esempio come (2d), dove sono già i tempi dei verbi, rispettivamente un trapassato prossimo e un passato prossimo, a indicare l'esatto ordine di svolgimento delle due azioni:

(4) a. Aveva regolarmente spedito (ieri) il plico, che è stato rispedito (oggi) al mittente b. È stato rispedito (oggi) al mittente il plico, che aveva regolarmente spedito (ieri) c. *Aveva regolarmente spedito (oggi) il plico, che è stato rispedito (ieri) al mittente d. *E stato rispedito (ieri) al mittente il plico, che aveva regolarmente spedito (oggi)

Alla fine, il legame morfologico tra due verbi reciproci stabilisce chiaramente non solo la marcatezza dell'elemento modificato (prefissato) rispetto a quello che presen-

11 Vd. in particolare Francescato (1996: 118-119) e ancora Vicario (c.s.). 
ta la semplice base verbale - l'abbiamo visto al $\S 2$ - ma anche l'ordine temporale, fattuale, nel quale i due elementi si presentano.

Passando da una coppia di verbi antonimi (reciproci) morfologicamente secondari a una coppia di verbi antonimi morfologicamente primari, viene naturalmente a mancare uno degli elementi decisivi dell'opposizione tra i due elementi (il prefisso). Ancora una volta, però, è possibile individuare un criterio per prevedere l'ordine in cui si presenteranno due verbi che indichino, rispettivamente, la variazione di uno stato, diciamo un allontanamento dalla posizione iniziale, e il ritorno allo stato, alla posizione, di partenza. $\grave{E}$ il caso, ad esempio, di verbi reciproci come andare vs. venire, salire vs. scendere, tirare vs. mollare:

(5) a. Il bidello andava e veniva dalla presidenza alla segreteria

b. I corrieri andavano e venivano dalla sede centrale alle filiali

c. Cristina aveva imparato in fretta a salire e scendere da cavallo

d. La quotazione dell'euro sale e scende ogni giorno

e. Con il tuo continuo tirare e mollare hai rotto la corda

f. Tirava e mollava sul prezzo della casa senza mai decidersi

Il significato di andare e venire presentati in successione, come in (5a) e (5b), va oltre quello della semplice somma dei due deittici e corrisponde, piuttosto, ad una sorta di 'andare avanti e indietro, muoversi senza sosta, spostarsi continuamente'. L'inversione dei due verbi produce un enunciato di significato piuttosto oscuro quanto meno ad una prima lettura - al limite dell'accettabilità (se non del tutto inaccettabile):

(6) a. ?Il bidello veniva e andava dalla presidenza alla segreteria

b. ?I corrieri venivano e andavano dalla sede centrale alle filiali

I due verbi paiono qui riportati al loro significato primario di descrivere un semplice moto a luogo (andare) e un moto da luogo (venire), precisando solamente il punto di vista dell'osservatore, che dipende naturalmente dalla rispettiva marca deittica. Così nella frase il bidello veniva e andava dalla presidenza alla segreteria, il riferimento alla posizione di partenza del soggetto e dell'osservatore sono ben definiti: il bidello si trova in presidenza e l'osservatore in segreteria. Con il bidello andava e veniva dalla presidenza alla segreteria, il soggetto può sì trovarsi in presidenza (ma anche in segreteria o altrove), e non viene specificata la posizione dell'osservatore.

Analoghe considerazioni si possono svolgere se si inverte l'ordine delle altre due coppie di verbi antonimi presentati in (5), salire e scendere o tirare e mollare:

(7) a. ${ }^{*}$ Cristina aveva imparato in fretta a scendere e salire da cavallo

b. ?La quotazione dell'euro scende e sale ogni giorno

c. *Per il continuo mollare e tirare, la corda si è rotta

d. ?Mollava e tirava sul prezzo della casa senza mai decidersi

Usando salire e scendere in senso letterale, quindi rispettivamente 'andare su, montare' e 'andare giù, smontare', l'enunciato (7a) risulta inaccettabile, non poten- 
dosi ragionevolmente immaginare che il soggetto (Cristina) si trovi abitualmente in sella al cavallo e che da questo prima scenda e poi salga: la posizione di partenza del soggetto deve essere senz'altro a terra e da qui si compirà prima l'operazione di salire a cavallo e quindi quella di scendervi. Nel caso di (7b), l'uso dei due verbi applicato ad un soggetto astratto (la quotazione di una moneta) rende l'enunciato più accettabile, ma ancora una volta un po' strano, descrivendo magari quella che risulta essere la normale oscillazione dell'euro che, per ipotesi, si deprezza all'apertura delle contrattazioni di borsa per poi riguadagnare valore nel proseguo della giornata ${ }^{12}$. Analogo discorso vale anche per tirare e mollare, nei due casi di (7c) e (7d), dove l'inversione dei verbi nell'uso letterale di (7c) risulta non ammissibile e appena accettabile in (7d).

L'ordine relativo assunto dai verbi antonimi finora esaminati, si nota ancora meglio se si considerano i sostantivi deverbali ottenuti dall'unione di questi elementi, rispettivamente andirivieni 'traffico, viavai, continuo andare e venire, confuso o irritante', saliscendi 'successione di tratti in salita e in discesa', anche 'sistema di chiusura di porte, imposte, battenti', e tiremmolla 'verificarsi di situazioni contrastanti tra loro', anche 'persona irresoluta, incerta'13.

(8) a. Come si fa a lavorare con questo andirivieni di gente?

b. In strada c'era un andirivieni di ambulanze e polizia

c. Questo sentiero è un continuo saliscendi

d. Per le scale c'era un bel saliscendi

e. Dopo un lungo tiremmolla abbiamo concluso l'affare

f. Quell'uomo è un tiremmolla, non decide mai niente

Nel caso di questi sostantivi, formati dall'unione di due verbi antonimi, si trova in prima posizione ancora l'elemento che trasmette l'indicazione di una variazione dello stato iniziale e in seconda posizione quello che esprime il ritorno allo stato precedente: un incremento di spazio, di distanza, e quindi una sua diminuzione è dato dalla coppia andare e venire (andirivieni, non *vieniandi, *vienievai), un aumento di una certa altezza e quindi un suo decremento si ha con salire e scendere (saliscendi, non *scendisali), una tensione e quindi un rilassamento si ha infine con tirare e mollare (tiremmolla, non * molletira) ${ }^{14}$.

Lo stesso ordine, collegato sempre con la successione aumento-diminuzione (quindi ancora con i tratti positivo-negativo) di spazio o di quantità in genere, si trova anche in altri verbi reciproci, come ad esempio comperare e vendere o aprire e chiudere:

12 Anche qui il significato di sale e scende, analogamente ad andirivieni, passa a rendere l'idea di un movimento oscillatorio, in generale, piuttosto che un vero e proprio moto di salita seguito da uno di discesa.

13

14 Molto interessante sarebbe verificare, in prospettiva tipologica, se questo ordine di elementi antonimi sia rispettato anche in altre lingue o quali siano i motivi che ne modificano, eventualmente, la successione. Immediato, a tale proposito, viene il controesempio dell'inglese come and go (piuttosto che * go and come), pur a un minore grado di lessicalizzazione dell'italiano andirivieni, ma anche dell'ungherese jövésmenés, ancora 'andirivieni'. 
(9) a. Al mercato, la gente comperava e vendeva di tutto

b. Comperare e vendere azioni in Borsa è il suo mestiere

c. A causa di un giro d'aria, la finestra si apriva e si chiudeva di continuo

d. Apre e chiude la bocca come per parlare, poi tace

Qui comperare, che indica l'aumento delle proprietà di chi compie l'azione, precede vendere (vi è per altro il sostantivo composto compravendita, non *vendicompera); aprire, che descrive l'aumento di spazio di comunicazione tra due luoghi (diversi) e percettivo in genere, precede chiudere ${ }^{15}$. Ancora, non è possibile dal punto di vista logico vendere qualcosa prima di averla comperata (ereditata, trovata o in qualche modo acquisita), come non pare possibile eseguire l'azione di chiudere su un oggetto in posizione non meglio specificata, assumendo quindi che a priori l'oggetto si trovi in stato di quiete, di chiusura (qui i casi riguardano una finestra e la bocca).

\section{Osservazioni conclusive}

Nella generale discussione sui caratteri propri del fenomeno dell'antonimia abbiamo svolto qui alcune brevi osservazioni sull'ordine in cui di norma si incontrano, in contesti non marcati, due elementi verbali di significato contrario presentati in sequenza. Si tratta di considerazioni che partono dal riconoscimento, all'interno di una coppia di verbi reciproci, di un elemento connotato come "positivo" (diciamo anche "progressivo"), che interviene a modificare un determinato stato, e di uno connotato come "negativo" (quindi "regressivo"), che tendenzialmente annulla l'effetto della prima azione e riconduce allo stato di partenza. Tale riconoscimento passa, in primo luogo, attraverso l'esame della struttura morfologica dei due verbi in opposizione. Se i due verbi appartengono alla categoria degli antonimi morfologici (o secondari), non sarà difficile assegnare al verbo di base, non modificato e quindi non marcato, il carattere di "positivo" e al suo contrario quello di "negativo": il verbo orientato al positivo predicherà infatti lo svolgimento di un'azione, l'altro la negazione, l'annullamento, dell'azione stessa. In questo caso, la sequenza dei due verbi risulterà positivo-negativo, non potendosi logicamente predicare l'annullamento di un'azione che non sia già compiuta: non è possibile disfare un lavoro che non sia già fatto, come non è possibile dischiudere una porta che non sia chiusa ${ }^{16}$. Analogo discorso vale anche per $\mathrm{i}$ verbi agentivi che predicano un moto proprio o figurato (di segno positivo) di un oggetto, come dare o portare, i quali avranno prefissati come ridare o riportare che indicano il ritorno (di segno negativo) dell'oggetto stesso alla posizione o allo stato di partenza.

Per un'analisi semantica di aprire e chiudere vd. Moneglia (1987: 295 sgg.).

16

Un caso particolare di questa opposizione, sul quale sarebbe comunque interessante tornare, è quella di verbi entrambi prefissati. Si considerino, ad esempio, il sostantivo colpa e i due denominali incolpare 'dare la colpa' e discolpare 'togliere la colpa': qui l'opposizione non riguarda un verbo con prefisso $\emptyset$ e uno con prefisso negativo, bensì uno con prefisso ingressivo e uno con prefisso negativo. 
Determinare l'ordine logico di verbi antonimi primari, nella frase, passa ancora attraverso la definizione di un elemento positivo e di uno negativo, all'interno della coppia, di uno che provoca un'alterazione dello stato di partenza e di un altro che ristabilisce le condizioni iniziali. Così tra i due verbi deittici, ad esempio, andare porterà un aumento della distanza dal parlante (positivo) e venire una sua diminuzione (negativo); allo stesso modo salire descriverà un aumento dell'altezza rispetto al punto di osservazione (positivo), scendere una diminuzione (negativo) e così via. Stabilire un determinato ordine positivo-negativo per alcuni verbi reciproci è sostenuto dall'esame dei sostantivi deverbali ottenuti dall'unione dei due elementi: è il caso di sostantivi italiani come andirivieni o saliscendi, che presentano lessicalizzato, e quindi stabilizzato, il rapporto logico e temporale tra le due azioni ${ }^{17}$. Il valore semantico di questi composti lessicalizzati, ne abbiamo fatto rapido cenno, supera in ogni caso la semplice somma dei significati dei due constituenti: sarà proprio in base alla trasparenza morfologica e semantica dei composti, cioè alla distanza del significato del composto lessicalizzato dalle basi di partenza, che se ne potrà stabilire, tra l'altro, il grado di lessicalizzazione.

In conclusione, la possibilità di verificare la posizione di verbi in opposizione antonimica, quando si trovino in sequenza, consente di discutere allo stesso tempo delle proprietà semantiche degli stessi. Questo esame, da affidare in ogni caso a lavori di ampia prospettiva tipologica e contrastiva, si fonda in buona sostanza sul principio di azione-reazione, dove descrivere un'azione di ritorno, diciamo negativa, implica una precedente variazione, positiva, dello stato di partenza.

17 Il valore semantico di questi composti lessicalizzati, ne abbiamo fatto rapido cenno, va comunque al di là della semplice somma dei significati dei due costituenti: sarà proprio in base alla trasparenza morfologica e semantica dei composti che se ne potrà stabilire, per altro, il grado di lessicalizzazione. 


\section{Bibliografia}

DINGS, J. 1986. Antonimia lessicale. "Quaderni di Semantica” 7, 2: 333-380.

DUCHÁCEK, O. 1965. Sur quelques problémes de l'antonymie. "Cahiers de Lexicologie" 6: 55-66.

FranCESCATo, G. 1996. Saggi di linguistica teorica e applicata. Torino, Dell’Orso.

GECKELER, H. 1979. La semantica strutturale. Torino, Boringhieri (tit. or. Strukturelle Semantik und Wortbildtheorie. München, Fink, 1971).

GECKELER, H. 1983. Observations sur l'absence de l'antonymie dans certaines sections du lexique. "Quaderni di Semantica" 4: 98-106.

ILIESCU, M. 1977. Oppositions sémantiques: antonymie linguistique et antonymie logique. "Folia Linguistica" 10: 151-168.

KLEIN, J. R. 1975. Adjectifs antonymiques et substantivation. "Cahiers de Lexicologie" 26: 47-55.

LEWIN, K. 1961. Principi di psicologia topologica. Firenze, Organizzazioni Speciali.

LYONS, J. 1971. Introduzione alla linguistica teorica. Bari, Laterza.

Lyons, J. 1980. Manuale di semantica. Bari, Laterza (tit. or. Semantics. I. Cambridge, University Press, 1977).

MONEGLIA, M. 1987. Senso e campi di variazione: una esplorazione sul significato di alcuni verbi causativi italiani. "Studi di Grammatica Italiana" 13: 271-349.

STATI, S. 1977. Il rapporto di antonimia. "Lingua e stile" 12: 385-404.

VICARIO, F. c.s. Antonimia verbale e morfologia: il prefisso ri- tra iterativo e reversivo. In: Atti del XXXVIII Congresso Internazionale di Studi della Società di Linguistica Italiana.

ZAMBONI, A. 1986. Considerazioni sull'it. andirivieni e sul relativo tipo composizionale. In: Lichem, K./Mara, E./Knaller, S. (a cura di). Parallela 2. Aspetti della sintassi dell'italiano contemporaneo. Tübingen, Narr: 329-341. 


\section{Povzetek}

\section{PARI POMENSKO NASPROTUJOČIH SI GLAGOLOV: BESEDNI RED}

Navedenih je nekaj opomb k vrstnemu redu glagolov $z$ nasprotujočim si pomenom $v$ sicer nezaznamovanem sobesedilu. Opazovanje izhaja iz prepričanja, da je mogoče pri taki dvojici glagolov prvega imeti za 'pozitivno', nekako 'progresivno' obarvanega, in ta obstoječe stanje spremeni, za drugega pa je značilna negativnost, 'regresivnost', torej težnja, da se izniči udejanjanje prvega dejanja in vzpostavi prvotno stanje. V članku se najprej tehta oblikoslovna zgradba obeh glagolov: če gre za glagola, za katera izvira nasprotovanje iz oblike, ne bo težko prvemu, osnovnemu glagolu, ki oblikovno ne kaže nobene spremembe in potemtakem ni označen, prisoditi karakter 'pozitivnega', onemu drugemu pa 'negativnega'. Prvi izraža razvoj neke dejavnosti, drugi pa nikalnost, izničenje akcije. $V$ tem primeru bo zaporedje obeh glagolov pozitivno-negativno, saj si ni mogoče zamisliti izničenja nekega dejanja, ki se še ni zgodilo; nekako tako, kot ni mogoče uničiti nekega dela, ki sploh ni bilo opravljeno, ali odpreti vrat, ki niso zaprta.

Če pa gre za primarno, pomensko opozicijo, je mogoče prepoznati v enem glagolu pozitivno, $\mathrm{v}$ drugem pa negativno prvino. Pri dvojici italijanskih glagolov kot andare 'iti, iti kam' in venire 'priti' izraža prvi večanje oddaljenosti od govorečega, drugi pa zmanjševanje take oddaljenosti. Podobno velja za dvojico salire 'povzpeti se' in scendere 'iti dol, spustiti se', pri čemer izraža prvi večanje višine glede na točko opazovanja, drugi pa zmanjševanje, torej nekako negativnost. Tak vrstni red se zdi časovno utemeljen in logičen; vsaj v italijanščini je ugotovljiv tudi pri samostalnikih, skovanih iz glagolskih oblik: andirivieni 'vrvež, beganje', saliscendi 'stalno vzpenjanje in spuščanje, npr. ceste'. 